(C) 2018 IEEE. Personal use of this material is permitted. Permission from IEEE must be obtained for all other uses, in any current or future media, including reprinting/republishing this material for advertising or promotional purposes, creating new collective works, for resale or redistribution to servers or lists, or reuse of any copyrighted component of this work in other works. 


\title{
Long-term functionality of a soft electrode array for epidural spinal cord stimulation in a minipig model
}

\author{
Giuseppe Schiavone, Fabien Wagner, Florian Fallegger, Xiaoyang Kang, Nicolas Vachicouras, \\ Beatrice Barra, Marco Capogrosso, Jocelyne Bloch, Grégoire Courtine, Stéphanie P. Lacour
}

\begin{abstract}
Long-term biointegration of man-made neural interfaces is influenced by the mechanical properties of the implant materials. Substantial experimental work currently aims at replacing conventional hard implant materials with soft alternatives that can favour a lower immune response. Here we assess the performance of a soft electrode array implanted in the spinal epidural space of a minipig model for a period of 6 months. The electrode array includes platinum-silicone electrode contacts and elastic thin-film gold interconnects embedded in silicone. In-vivo electrode impedance and voltage transients were monitored over time. Following implantation, epidural stimulation produced muscle-specific evoked potentials and visible muscle contractions. Over time, postoperative and stimulation induced changes in electrode impedance were observed. Such trends provide a basis for future technological improvements aiming at ensuring the stability of soft implantable electrodes for neural interfacing.
\end{abstract}

\section{INTRODUCTION}

Epidural Electrical Stimulation (EES) of the spinal cord is a well-established neuromodulation technique that is employed nowadays in the clinic for the relief of chronic pain [1], [2], and in locomotor rehabilitation research after Spinal Cord Injury (SCI), both in animal models [3], [4] and human subjects [5], [6]. The spinal cord is surrounded by a heterogeneous mechanical environment that imposes challenging requirements on implanted devices in order to comply with the physiological range of movement and deformation of the different biological structures. Although the introduction of microtechnology has in recent years revolutionised the field of medical devices, enabling researchers and manufacturers to develop smart multimodal implantable systems, the stability of the interfaces created between man-made implants and the target biological structures tend to suffer from instability over long implantation periods of several months. One of the key

G. Schiavone, F. Fallegger, X. Kang, N. Vachicouras, and S. P. Lacour are with the Center for Neuroprosthetics, School of Engineering, Institutes of Microengineering and Bioengineering, Ecole Polytechnique Federale de Lausanne (EPFL), Lausanne, Switzerland (e-mail: giuseppe.schiavone@epfl.ch, xiaoyang.kang@epfl.ch,

stephanie.lacour(epfl.ch). florian.fallegger@epfl.ch, nicolas.vachicouras@epfl.ch,

B. Barra and M. Capogrosso are with the Department of Neuroscience and Movement Science, Platform of Translational Neuroscience, University of Fribourg. Fribourg, Switzerland (e-mail: beatrice.barra@unifr.ch, marco.capogrosso@unifr.ch ).

F. Wagner and G. Courtine are with the Center for Neuroprosthetics and Brain Mind Institute, School of Life Sciences, Ecole Polytechnique Federale de Lausanne (EPFL), Lausanne, Switzerland (e-mail: fabien.wagner@epfl.ch, gregoire.courtine (a)epfl.ch).

J. Bloch is with the Centre Hospitalier Universitaire Vaudois (CHUV), Lausanne, Switzerland (e-mail: jocelyne.bloch@chuv.ch). factors behind such instability is the large difference in mechanical properties between materials conventionally used to fabricate electronics, typified by silicon $(\sim 150 \mathrm{GPa})$ and the host biological tissue $(0.1-3 \mathrm{kPa})$ [7]. As the socalled mechanical mismatch has been shown to trigger foreign body reaction in the host [8], [9], researchers have since resorted to adapting existing technologies to softer device materials [10]-[12].

We have previously developed a manufacturing toolkit based on soft and stretchable technology for the fabrication of compliant devices that can be deployed in contact with neural tissue without triggering significant foreign body reaction [13]. Here, we report on a preliminary experiment conducted on a large animal model (adult Göttingen minipig, $80 \mathrm{~kg}$ weight), aimed at assessing the long-term functionality of implants based on our existing soft neurotechnology. An 8-polar electrode array was implanted dorsally in the lumbar epidural space at a location that enables the recruitment of hind-limb muscles by means of electrical stimulation. The functionality of the implant was tested with stimulation tests and impedance measurements in-vitro, intra-operatively, then at 1,3 , and 6 months (36, 86, and 186 days) postimplantation, and ex-vivo.

\section{METHODS}

\section{A. Fabrication of the electrode array}

An 8-electrode array implant was fabricated in a cleanroom environment by embedding gold thin-film tracks (55 nm thick) between two layers of silicone rubber (DowCorning Sylgard 184 polydimethylsiloxane, PDMS), for a total stack thickness of about $0.5 \mathrm{~mm}$. The top silicone layer includes vias that create 8 active electrode sites, through which a soft coating is screen printed onto the gold film, with a geometric surface area of about $2 \mathrm{~mm} \times 0.7 \mathrm{~mm}$. This electroactive material is prepared by dispersing meso-scale platinum particles (Goodfellow Cambridge Ltd. PT006021 platinum powder, max. particle size $3.5 \mu \mathrm{m}$, purity $99.95 \%$ ) in a PDMS matrix, in order to create a conductive paste that offers a balance between the charge injection properties of platinum and the mechanical properties of PDMS. Each gold thin-film track is then connected to a wire interfaced with a drop of conductive silver epoxy (EPO-TEK ${ }^{\circledR}$ H27D part A). The contacts between the wires and the tracks are finally encapsulated with a room-temperature curing sealant (one component silicone sealant 734, Dow Corning) and bundled in a cable. Before implantation, the device is sterilised with an ethylene oxide (ETO) treatment.

\section{B. Surgical procedures}

The experimental protocol was approved by the cantonal 
(Vaud) and federal (Swiss) veterinary authorities (authorisation no. VD3117). Deep anaesthesia was induced with intramuscular injection of ketamine/xylazine and maintained with 1-3\% isoflurane after intubation. Heart rate, body temperature and blood oxygenation were constantly monitored. A dorsal mid-line skin incision was made from vertebral level L1 to L5, and the muscles covering the laminae were retracted. A complete flavectomy (removal of the ligament) was performed at vertebral level L4/L5. Partial midline flavectomies were performed at levels L3/L4, L2/L3 and L1/L2. A soft silicone guiding tool was inserted epidurally following the midline, from L4/L5 to L1/L2. The tip of the array implant was anchored to the tail of the guiding tool, which was pulled to slide the electrodes into the epidural space, following the prepared path. The 8-polar cable was looped in the tissue and the connector secured subcutaneously for easy access during subsequent acute measurement sessions.

To record electromyographic (EMG) activity, bipolar electrodes were prepared by trimming a small section of insulation from Teflon-coated stainless steel wires (AS631, Cooner Wire). Three pairs of electrode wires plus a ground wire were tunnelled from the location of the target muscles through to the mid-line skin incision already made in the back to access the spinal cord. Three muscles of the right leg were implanted: semitendinosus (ST, EMG 1), tibialis anterior (TA, EMG 2), and vastus lateralis (VL, EMG 3). The EMG electrodes were connected to a percutaneous connector (A79110-001, Omnetics Connector Corporation), which was surgically placed in a position adjacent to the 8polar connector of the spinal array.

Fig. 1 shows diagrams and photographs of the implanted spinal array and EMG electrodes. After taking intraoperative measurements, the skin incision on the back of the animal was closed with 3 suturing layers (fascia, subcutaneous and skin). Acute measurement tests were taken at time points of 36,86 , and 186 days post-implantation by partially reopening the skin and subcutaneous tissue (under isoflurane anaesthesia) to expose the array and EMG connectors. At the end of each acute measurement, the incision was closed again with 2 suturing layers (subcutaneous and skin).

\section{Soft electrode array characterisation}

Before implantation, in-vitro measurements were taken by immersing the array under test in phosphate buffered saline solution (Gibco PBS, pH 7.4, 1X), along with a counter $(\mathrm{Pt}$ mesh) and reference electrode (Metrohm, El. Ag/AgCl DJ RN SC: $\mathrm{KCl}$ ). In this configuration, Voltage Transients (VT) following constant current pulsing [14] were measured to assess the charge injection properties of the 8 electrodes. Current-controlled, symmetric, biphasic, charge balanced, cathodic-first pulses are applied between the electrode under test (working electrode) and the counter with an A-M Systems 2100 Isolated Pulse Stimulator $(300 \mu \mathrm{s} /$ phase pulse width, $1 \mathrm{~s}$ inter-pulse period, $1 \mathrm{~mA}$ and $2 \mathrm{~mA}$ amplitude), while measuring the voltage across the working and reference electrodes with an oscilloscope. The minimum voltage recorded is taken (in absolute value) as a metric indicating the voltage required to inject a cathodic current pulse with the chosen amplitude.

Electrochemical Impedance Spectroscopy (EIS) measurements using a three-electrode setup were also taken using a Gamry 600 potentiostat to characterise the electrochemical properties of the electrodes $(50 \mathrm{mV}$ amplitude, $1 \mathrm{~Hz}-1 \mathrm{MHz}$ frequency).

Analogous measurements have been taken in-vivo using the separate contacts of the implanted array as working electrodes and a subdermal needle inserted in the dorsal skin, acting both as counter and reference electrode (2electrode system). Identical stimulation parameters are applied in-vitro and in-vivo.

\section{Muscle response and recruitment curves}

Single EES pulses were delivered to the spinal cord through 3 different electrodes on the spinal array (electrodes 1,2 , and 3 , caudal to rostral respectively), eliciting motor response in the 3 muscles implanted with EMG electrodes. For each electrode, we visually identified the minimum amplitude necessary to elicit a motor response (referred hereafter as motor threshold) and the amplitude at which all 3 motor evoked potentials were saturated (referred hereafter as saturation amplitude). In separate runs, trains of about 60 stimulation pulses were delivered at about $1 \mathrm{~Hz}$ (amounting to about 1 minute of total stimulation time) and constant amplitude, while recording the EMG activity. This procedure was repeated for stimulation amplitudes ranging from motor threshold to saturation, and across the 3 selected array electrodes.

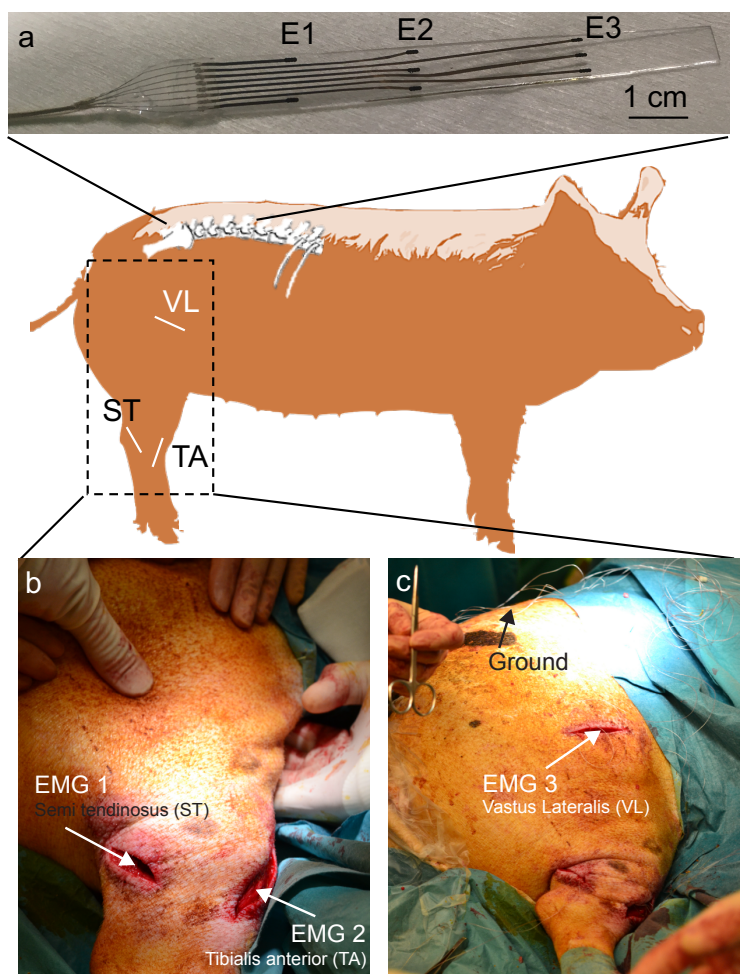

Fig. 1. Implantation diagram for the spinal electrode array and EMG electrodes. The array (a, top view) is inserted in the lumbar epidural space to electrically recruit muscles in the hind-limb. The three muscles selected for EMG recordings are the right semitendinosus (ST), tibialis anterior (TA), and vastus lateralis (VL) (b, c). 
The recorded EMG signals were band-pass filtered $(30 \mathrm{~Hz}$ - $800 \mathrm{~Hz}$ ), sampled at $6 \mathrm{kHz}$, and normalised to the maximum activity recorded across all the responses obtained by stimulating from a specific electrode of the spinal array. The muscle recruitment level $\mu$ was evaluated by calculating the integral of the rectified triggered EMG signals immediately following the stimulation pulse. Recruitment curves were built by plotting the level of muscle recruitment as a function of the amplitude of the stimulation current.

\section{RESULTS}

\section{A. Time evolution of the electrical properties of electrodes}

VT measurements taken on all 8 electrodes at the different time points during the implantation period reveal that it was possible, with the equipment used, to inject $2 \mathrm{~mA}$ current pulses through 5 of the 8 implanted electrodes (Fig. 2) up to 6 months post-implantation. In-vitro, the voltage required to inject current pulses was found to be about $1.1 \mathrm{~V}$ and $1.9 \mathrm{~V}$ for $1 \mathrm{~mA}$ and $2 \mathrm{~mA}$ pulses, respectively. When recorded intra-operatively, the minimum negative voltages increased to $5.7 \mathrm{~V}$ and $9.3 \mathrm{~V}$ and kept drifting over time, indicating possible deterioration of the implant and/or evolution of the electrode-tissue interface. At day 182, the voltage for $1 \mathrm{~mA}$ stimulation pulses showed a drop compared to the previous data point. Although only 3 electrodes were stimulated with $1 \mathrm{~mA}$ in this session, this trend was also confirmed by subsequent impedance measurements. This was not observed however for stimulation at $2 \mathrm{~mA}$. After explantation, current pulsing was possible both at $1 \mathrm{~mA}$ and $2 \mathrm{~mA}$ on 6 out of the 8 implanted electrodes.

Not all electrodes could be tested in all runs due to limits in the total duration of the anaesthesia or too strong muscle contractions elicited at these current levels.

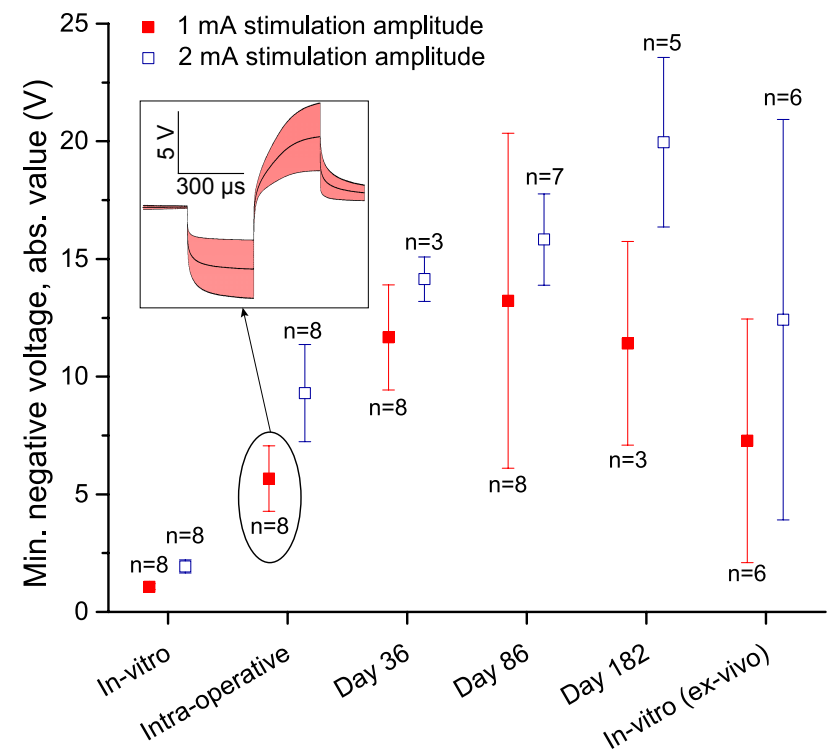

Fig. 2. Evolution of the minimum negative voltage over the implantation period for $1 \mathrm{~mA}$ and $2 \mathrm{~mA}$ pulses. The data points are averages and the error bars are standard deviations. The labels next to each data point indicate the number of electrodes measured. The inset shows the VT plot for one of the time points, taken at $1 \mathrm{~mA}$ stimulation current: the curve is the voltage response (average of 8 electrodes), and the shaded area represents the standard deviation.
The evolution over time of the impedance modulus at $1 \mathrm{kHz}$ of the measured electrodes is shown in Fig. 3. After an immediate increase from an average of about $0.7 \mathrm{kOhm}$ in-vitro to $19.55 \mathrm{kOhm}$ in-vivo, the interface impedance ramped up during the first month of implantation to about $153 \mathrm{kOhm}$ and then down to about $61 \mathrm{kOhm}$ on the last measurement session. This trend reflects what was observed with the VT measurements at $1 \mathrm{~mA}$ stimulation (Fig. 2).

The measurements taken in-vitro post-explantation revealed that permanent material degradation might have occurred that affects the electrochemical properties of the electrodes, such as damage to the electrode coating and increased interconnect resistance.

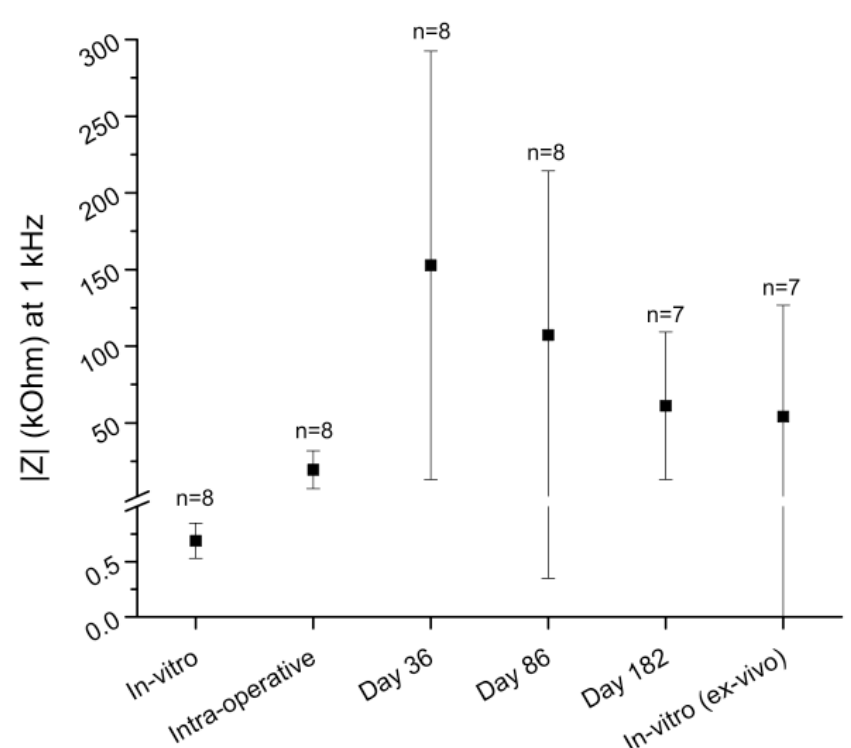

Fig. 3. Evolution of the modulus of the electrochemical impedance at $1 \mathrm{kHz}$ of the array electrodes over the implantation period. The data points are averages and the error bars are standard deviations. The labels next to each data point indicate the number of electrodes measured.

\section{B. Motor potentials following EES}

Fig. 4 shows an example of muscle recruitment from one of the spinal electrodes. Intra-operatively, electrode 2 on the array activated the implanted muscles with the recorded evoked potentials shown in Fig. 4 (top-left). One month post-implantation, no substantial change in the recruitment pattern was observed on this electrode, as illustrated by the curves in Fig. 2 (top-right and bottom-left). Three months post-implantation, however the recruitment of the muscles was considerably increased for the TA and VL, and reduced for the ST. Six months post-implantation, current pulses in similar amplitude range could still be delivered with electrode 2, but no EMG response was observed. This change, coupled with similar observations on electrodes 1 and 3, suggested that the array could have moved within the epidural space to a position that did not elicit motor responses, or that build-up of scar tissue in the chronic phase between 3 and 6 months could have prevented motor responses to be elicited by EES. 
Motor potentials (intra-op)

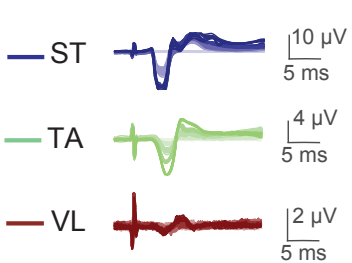

Recruitment curve (E2, intra-op)

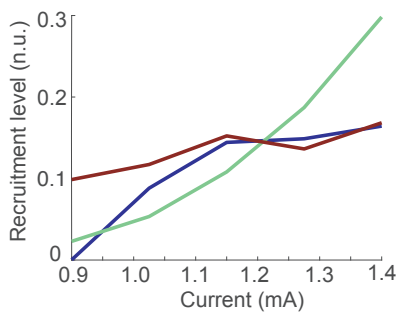

Recruitment curve (E2, 1 month)

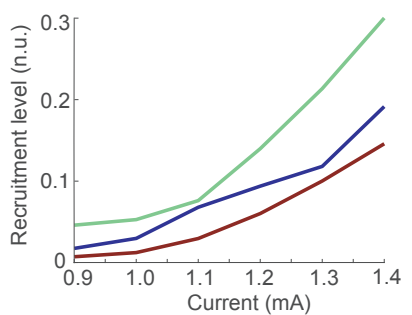

Recruitment curve (E2, 3 months)

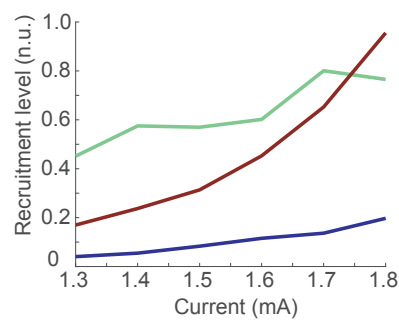

Fig. 4. Examples of muscle recruitment over time: intra-operatively recorded motor potentials for the 3 muscles using spinal electrode 2 (topleft, colour shades indicate different stimulation amplitudes); recruitment curves measured intra-operatively (top-right), and 1 and 3 months postimplantation (bottom left and right, respectively). Muscle recruitment is expressed in normalised units.

\section{Discussion AND CONCLUSIONS}

The results presented in this study show complete functionality of the implanted array until 3 months postimplantation. The voltage transient measurements shown in Fig. 2 demonstrate the delivery of stimulation pulses of up to $2 \mathrm{~mA}$ ( $300 \mu$ s pulse width) with 5 out of the 8 electrodes of the implanted device 6 months post-implantation, while impedance measurements taken in-vivo and shown in Fig. 3 indicate that 7 electrodes could still be interrogated.

The voltage required to inject the prescribed stimulation pulses in-vivo increased significantly over the course of the implantation period. In a lab environment, this can be accounted for with a high-voltage external stimulator. However, in the context of a long-term neuroprosthetic device, implanted pulse generators restrict the maximum available voltage for stimulation. Besides this purely electrical requirement, ensuring a low-impedance interface is important in order to maintain voltages within safe stimulation ranges [15]. Further work is therefore required to develop chronically stable low-impedance coatings that can guarantee safe charge injection in-vivo on the long-term.

EMG activity was recorded chronically up to 3 months post-implantation, although with varying muscle activation patterns. The changes in the motor responses indicate that the implant position could have progressively shifted from its original placement, and/or that possible build-up of scar tissue between the electrodes and the spinal cord could have prevented stable muscle recruitment.

The complete collection of data measured in-vivo indicates potential for all-soft electrode arrays as chronic neural interfaces. The trends observed in the stimulation tests suggest, however, that the adaptation of existing technologies for use in different animal models involves technological and clinical challenges that cannot be easily predicted from lab bench experiments. From a translational point of view, further work is therefore required to reach a more detailed understanding of the mechanical, electrochemical and biological mechanisms that affect the reliability of soft implants deployed chronically in large animals. These are necessary steps that, accompanied by adequate comparison with conventional technologies and tissue staining, will feed back into design and manufacturing with the aim of producing implants that can ensure longlasting biointerfacing.

\section{ACKNOWLEDGMENTS}

This work is supported by: the European Union's Horizon 2020 research and innovation programme under the Marie Skłodowska-Curie grant agreement No. 665667; the SNF Sinergia grant CRSII3_160696/1; the Wyss Center for Bio and Neuro Engineering; the Bertarelli foundation. The authors would like to thank Marco Burki for his precious help in the preparation of the animal and surgery room.

\section{REFERENCES}

[1] N. M. M.D, 'Spinal Cord Stimulation for Chronic Back and Neck Pain', Spine-health. [Online]. Available: https://www.spinehealth.com/treatment/back-surgery/spinal-cord-stimulation-chronicback-and-neck-pain. [Accessed: 11-Feb-2018]

[2] A. K. Compton, B. Shah, and S. M. Hayek, 'Spinal Cord Stimulation: A Review', Curr. Pain Headache Rep., vol. 16, no. 1, pp. 35-42, Feb. 2012.

[3] V. K. Mushahwar, D. F. Collins, and A. Prochazka, 'Spinal Cord Microstimulation Generates Functional Limb Movements in Chronically Implanted Cats', Exp. Neurol., vol. 163, no. 2, pp. 422 429, Jun. 2000

[4] D. Barthélemy, H. Leblond, J. Provencher, and S. Rossignol, 'Nonlocomotor and Locomotor Hindlimb Responses Evoked by Electrical Microstimulation of the Lumbar Cord in Spinalized Cats', J. Neurophysiol., Dec. 2006.

[5] K. Minassian et al., 'Stepping-like movements in humans with complete spinal cord injury induced by epidural stimulation of the lumbar cord: electromyographic study of compound muscle action potentials', Spinal Cord, vol. 42, no. 7, p. 401, Jul. 2004.

[6] M. R. Dimitrijevic, Y. Gerasimenko, and M. M. Pinter, 'Evidence for a Spinal Central Pattern Generator in Humansa', Ann. N. Y. Acad. Sci., vol. 860, no. 1, pp. 360-376, Nov. 1998.

[7] S. Wagner and S. Bauer, 'Materials for stretchable electronics', MRS Bull., vol. 37, no. 3, pp. 207-213, Mar. 2012.

[8] K. Franze, P. A. Janmey, and J. Guck, 'Mechanics in Neuronal Development and Repair', Annu. Rev. Biomed. Eng., vol. 15, no. 1, pp. 227-251, 2013.

[9] D. E. Discher, P. Janmey, and Y.-L. Wang, 'Tissue cells feel and respond to the stiffness of their substrate', Science, vol. 310, no. 5751, pp. 1139-1143, Nov. 2005.

[10] S. I. Park et al., 'Soft, stretchable, fully implantable miniaturized optoelectronic systems for wireless optogenetics', Nat. Biotechnol., vol. 33 , no. 12 , p. 1280 , Dec. 2015

[11] A. Altuna et al., 'SU-8 based microprobes for simultaneous neural depth recording and drug delivery in the brain', Lab. Chip, vol. 13, no. 7, pp. 1422-1430, Mar. 2013

[12] D.-H. Kim et al., 'Dissolvable films of silk fibroin for ultrathin conformal bio-integrated electronics', Nat. Mater., vol. 9, no. 6, p. 511, Jun. 2010.

[13] I. R. Minev et al., 'Electronic dura mater for long-term multimodal neural interfaces', Science, vol. 347, no. 6218, pp. 159-163, Jan. 2015.

[14] S. F. Cogan, 'Neural stimulation and recording electrodes', Annu. Rev. Biomed. Eng., vol. 10, pp. 275-309, 2008.

[15] T. L. Rose and L. S. Robblee, 'Electrical stimulation with $\mathrm{Pt}$ electrodes. VIII. Electrochemically safe charge injection limits with $0.2 \mathrm{~ms}$ pulses (neuronal application)', IEEE Trans. Biomed. Eng., vol. 37, no. 11, pp. 1118-1120, Nov. 1990. 